\title{
Vibrational contributions to static linear and nonlinear optical coefficients: from two-level to two-band systems
}

\author{
Chia-Chun Chou $\cdot$ Bih-Yaw Jin
}

Received: 28 October 2008/ Accepted: 17 January 2009/Published online: 10 February 2009

(C) Springer-Verlag 2009

\begin{abstract}
The importance of vibrational contributions to the static linear and nonlinear optical coefficients is investigated. We apply the exact sum-over-state (SOS) formulas for polarizabilities and hyperpolarizabilities expressed in terms of vibronic states to a two-level system with a single vibrational mode. The Herzberg-Teller expansion is applied to the SOS formulas including vibrational energy levels without employing the Placzek's approximation within both the Born-Oppenheimer approximation and electrical and mechanical harmonicities. The results include not only the vibrational contribution from the lattice relaxation expression but also the contribution arising from the higher-order correction terms. Model calculations on a diatomic system with two electronic states show that the contribution of these correction terms is small. Moreover, most of these higher-order terms are negligible in the solidstate limit. In polyacetylene, the contribution of the lattice relaxation expression is much larger than that in the diatomic case. Within the tight-binding approximation, the contribution of the lattice relaxation expression is $44 \%$ of the pure electronic contribution for the second hyperpolarizability.
\end{abstract}

C.-C. Chou · B.-Y. Jin $(\bowtie)$

Department of Chemistry, Center of Theoretical Sciences, Center of Quantum Science and Engineering, National Taiwan University, Taipei 10617, Taiwan, ROC

e-mail: byjin@ntu.edu.tw

Present Address:

C.-C. Chou

Department of Chemistry and Biochemistry, Institute for

Theoretical Chemistry, The University of Texas at Austin, Austin, TX 78712, USA
Keywords Nonlinear optical properties . Hyperpolarizabilities · Vibrational contributions . Polyenes

\section{Introduction}

There has been increasing interest in the study of nonlinear polarizabilities motivated greatly by the potential for using this property to design optical communication devices. The design of materials with large optical nonlinearities is an active and well-reviewed area of research [1]. Nonlinear optical processes are governed by molecular hyperpolarizabilities. These properties can be divided into contributions originating from the effects of electric fields on electronic motion and on nuclear motion. There has been a growing number of calculations of vibrational polarizabilities and hyperpolarizabilities in the past decades [2-10]. Polarizabilities and hyperpolarizabilities can be defined by a perturbation theory treatment of the electric fields, and this produces the sum-over-state (SOS) formulas in terms of vibronic energies and dipole moment matrix elements. The effect of including vibrational levels into the SOS expressions for polarizabilities and hyperpolarizabilities has been examined under the electrical and mechanical harmonicity approximation, and the perturbation formulas including corrections for mechanical and electrical anharmonicities have been derived [3-5].

Several approaches have been introduced to calculate vibrational hyperpolarizabilities resulting from the effects of anharmonicity. Restricted Hartree-Fock 6-31G calculations have been performed to determine electrical and mechanical anharmonicity contributions to the longitudinal vibrational second hyperpolarizability for several 
conjugated oligomers [11]. Field-induced coordinates have been used to describe the vibrational polarizability and hyperpolarizabilities due to nuclear relaxation [12]. The effect of basis set and electron correlation beyond the MP2 method on the electronic and vibrational hyperpolarizabilities has been investigated [13]. Moreover, a variational approach has been developed to calculate vibrational linear and nonlinear optical properties of molecules with large electrical and mechanical anharmonicities $[14,15]$.

On the other hand, a semiclassical treatment has been used to derive an explicit analytical expression for vibrational contribution in terms of spectroscopic observables [2]. The lattice relaxation expression has been obtained from the exact SOS formulas within the Placzek's approximation [4]. In addition, the lattice relaxation expression has been utilized to discuss nonlinear optical properties of charge-transfer organic materials in the framework of the valence-bond charge-transfer model [16-23].

The purpose of this paper is to derive the lattice relaxation expression from the SOS formulas by including the vibrational energies in the denominators of the SOS formulas without employing the Placzek's approximation. We apply the exact SOS formulas for the (hyper)polarizabilities expressed by vibronic states to a two-level system with a single vibrational mode. The vibrational mode is described by the displaced harmonic oscillator model. Within the double harmonic approximation (electrical and mechanical harmonicities), we obtain the lattice relaxation expression for the vibrational contribution. Furthermore, analytical expressions for the contributions arising from the next higher-order correction terms are also derived through the Herzberg-Teller expansion. The importance of the various terms is examined for ethylene and polyacetylene. Model calculations show that the contribution from these higher-order terms is relatively small for ethylene. In addition, most of the contributions from the higher-order terms are negligible in the solidstate limit. The contribution from the lattice relaxation expression in polyacetylene is much larger than that in the diatomic case. Within the tight-binding approximation, the contribution of the lattice relaxation expression is $44 \%$ of the pure electronic contribution for the second hyperpolarizability.

The paper is organized as follows: we begin by considering a two-level system with a single vibrational mode, and use the exact SOS formulas to derive analytical expressions for the static (hyper)polarizabilities in Sect. 2. In Sect. 3, contributions from the various terms in the polarizability and the second hyperpolarizability are calculated for ethylene and infinite polyene. Finally, we make some comments and conclude with a discussion about future research in Sect. 4.

\section{Two-level system with a single vibrational mode}

A two-level system with a single vibrational mode is considered. The vibrational levels of the ground and excited electronic states are modeled by harmonic oscillators (the mechanical harmonicity approximation). For simplicity, these oscillators are assumed to have identical frequencies $\omega$, but minima for the ground electronic state and for the excited electronic state are displaced. The energy difference between the minima of the two electronic states is denoted by $\Delta$.

\subsection{Static polarizability}

In the presence of an external electromagnetic field $\varepsilon$, the interaction energy between the molecules and the field is given by

$E^{\text {int }}=-\mu \varepsilon-\frac{\alpha}{2 !} \varepsilon^{2}-\frac{\beta}{3 !} \varepsilon^{3}-\frac{\gamma}{4 !} \varepsilon^{4}-\cdots$.

Therefore, the total energy becomes

$E=E_{0}+E^{\text {int }}=E_{0}-\mu \varepsilon-\frac{\alpha}{2 !} \varepsilon^{2}-\frac{\beta}{3 !} \varepsilon^{3}-\frac{\gamma}{4 !} \varepsilon^{4}-\cdots$,

where $E_{0}$ is the energy at zero field, $\mu$ is the dipole moment, $\alpha$ is the polarizability, and $\beta$ and $\gamma$ are hyperpolarizabilities.

From the perturbation theory, the polarizability $\alpha$ is expressed by

$\frac{1}{2 !} \alpha=\alpha^{\prime}=\sum_{m \neq 0} \frac{\langle 0|\mu| m\rangle\langle m|\mu| 0\rangle}{E_{m}-E_{0}}$.

From this equation, we obtain the polarizability for a twolevel system with a single vibrational mode

$\alpha^{\prime}=\sum_{m \neq 0} \frac{|\langle g 0|\mu| g m\rangle|^{2}}{E_{g m}-E_{g 0}}+\sum_{\bar{m}=0} \frac{|\langle g 0|\mu| e \bar{m}\rangle|^{2}}{E_{e \bar{m}}-E_{g 0}}$.

The bar over the $m$ in the $|\bar{m}\rangle$ indicates that this is a vibrational level of the excited electronic state, and $|m\rangle$ denotes the vibrational levels of the ground state. Within the Born-Oppenheimer approximation, we can write the dipole moment of the ground electronic state as a function of vibrational coordinate, and the matrix elements in the first term in Eq. 3 becomes

$\langle g 0|\mu| g m\rangle=\left\langle 0\left|\mu_{g g}(Q)\right| m\right\rangle$.

We choose the minimum for the ground electronic state to be zero and expand the electronic dipole moment in a 
Taylor series around the minimum. Within the electrical harmonicity approximation, the dipole moment is expanded to the first derivative term

$\mu_{g g}(Q)=\mu_{g g}(0)+\left(\frac{\partial \mu_{g g}}{\partial Q}\right)_{0} Q$.

Substituting Eqs. 4 and 5 into Eq. 3, we obtain

$\alpha^{\prime}=\frac{1}{2 k}\left(\frac{\partial \mu_{g g}}{\partial Q}\right)_{0}\left(\frac{\partial \mu_{g g}}{\partial Q}\right)_{0}+\sum_{\bar{m}=0} \frac{|\langle g 0|\mu| e \bar{m}\rangle|^{2}}{\Delta+\bar{m} \hbar \omega}$,

where $k$ is the force constant of the ground electronic state and $|\langle 0|Q| 1\rangle|^{2}=\hbar / 2 m \omega$ has been used.

The first term in Eq. 6 is the lattice relaxation expression for vibrational contribution to the polarizability. If the vibrational frequencies are much smaller than the electronic frequencies $(\hbar \omega \ll \Delta)$, the first term will dominate. Moreover, this term arises completely from the pure vibrational contribution to the polarizability because no electronic excitation is involved. However, the contribution expressed by the second term in Eq. 6 results from coupled motion of the electronic excitation and nuclear vibration within the adiabatic approximation.

Instead of using the Placzek's approximation, we expand the second term in Eq. 6 in powers of $\hbar \omega / \Delta$

$$
\begin{aligned}
\sum_{\bar{m}=0} \frac{|\langle g 0|\mu| e \bar{m}\rangle|^{2}}{\Delta+\bar{m} \hbar \omega}= & \frac{1}{\Delta} \sum_{\bar{m}=0}\left\langle 0\left|\mu_{g e}\right| \bar{m}\right\rangle\left\langle\bar{m}\left|\mu_{e g}\right| 0\right\rangle \\
& \times\left[1-\bar{m} \frac{\hbar \omega}{\Delta}+\bar{m}^{2}\left(\frac{\hbar \omega}{\Delta}\right)^{2}-\cdots\right] .
\end{aligned}
$$

Here, we assume that the electronic transition moment $\mu_{g e}(Q)$ is real valued. Since the vibrational levels of the excited state $|\bar{m}\rangle$ form a complete set, the first term in Eq. 7 can be reduced to

$\sum_{\bar{m}=0}\left\langle 0\left|\mu_{g e}\right| \bar{m}\right\rangle\left\langle\bar{m}\left|\mu_{g e}\right| 0\right\rangle=\left\langle 0\left|\mu_{g e}^{2}\right| 0\right\rangle$.

We also write the electronic transition moment as a function of the vibrational coordinate and expand it in a Taylor series around the minimum of the ground electronic state to the first derivative term

$\mu_{g e}(Q)=\mu_{g e}(0)+\left(\frac{\partial \mu_{g e}}{\partial Q}\right)_{0} Q$

Substituting Eq. 9 into Eq. 8, we obtain

$\left\langle 0\left|\mu_{g e}^{2}\right| 0\right\rangle=\mu_{g e}^{2}(0)+\left(\frac{\partial \mu_{g e}}{\partial Q}\right)_{0}^{2} \frac{\hbar}{2 m \omega}$.

Similarly, substituting Eq. 9 into the second term on the right side of Eq. 7 yields

$$
\begin{aligned}
& \sum_{\bar{m}=0} \bar{m}\left\langle 0\left|\mu_{g e}\right| \bar{m}\right\rangle\left\langle\bar{m}\left|\mu_{e g}\right| 0\right\rangle=\sum_{\bar{m}=0} \bar{m}\left[\mu_{g e}^{2}(0)\langle 0 \mid \bar{m}\rangle\langle\bar{m} \mid 0\rangle\right. \\
& \quad+\mu_{g e}(0)\left(\frac{\partial \mu_{g e}}{\partial Q}\right)_{0}(\langle 0 \mid \bar{m}\rangle\langle\bar{m}|Q| 0\rangle+\langle 0|Q| \bar{m}\rangle\langle\bar{m} \mid 0\rangle) \\
& \left.\quad+\left(\frac{\partial \mu_{g e}}{\partial Q}\right)_{0}^{2}\langle 0|Q| \bar{m}\rangle\langle\bar{m}|Q| 0\rangle\right] .
\end{aligned}
$$

It is noted that the first term is related to the FranckCondon factor for the displaced harmonic oscillator model

$I=|\langle 0 \mid \bar{m}\rangle|^{2}=\frac{S^{\bar{m}} e^{-S}}{\bar{m} !}$.

Hence, this term can be simplified by

$\sum_{\bar{m}=0}^{\infty} \bar{m}\langle 0 \mid \bar{m}\rangle\langle\bar{m} \mid 0\rangle=S$,

where $S$ is the Huang-Rhys factor. Through the raising and lowering operator formalism of the harmonic oscillator, the second term in Eq. 11 becomes

$$
\begin{aligned}
& \sum_{\bar{m}=0}^{\infty} \bar{m}(\langle 0 \mid \bar{m}\rangle\langle\bar{m}|Q| 0\rangle+\langle 0|Q| \bar{m}\rangle\langle\bar{m} \mid 0\rangle) \\
& =2 \sqrt{\frac{\hbar}{2 m \omega}} \sum_{\bar{m}=0}^{\infty} \bar{m}\langle 0 \mid \bar{m}\rangle\langle\bar{m} \mid 1\rangle
\end{aligned}
$$

The closed form of the summation in Eq. 14 has been obtained [24, 25]; hence, the second term in Eq. 11 becomes

$$
\begin{aligned}
& \sum_{\bar{m}=0}^{\infty} \bar{m}(\langle 0 \mid \bar{m}\rangle\langle\bar{m}|Q| 0\rangle+\langle 0|Q| \bar{m}\rangle\langle\bar{m} \mid 0\rangle) \\
& =2 \sqrt{\frac{\hbar}{2 m \omega}}\left(-\frac{\delta}{2}\right)
\end{aligned}
$$

where $\delta$ serves as a dimensionless measure of the displacement $d$ of the oscillators $\delta=d / \sqrt{\left\langle 0\left|Q^{2}\right| 0\right\rangle}$. In the same way, we obtain the third term in Eq. 11

$$
\sum_{\bar{m}=0}^{\infty} \bar{m}\langle 0|Q| \bar{m}\rangle\langle\bar{m}|Q| 0\rangle=\frac{\hbar}{2 m \omega} \sum_{\bar{m}=0}^{\infty} \bar{m}|\langle 1 \mid \bar{m}\rangle|^{2}=\frac{\hbar}{2 m \omega}(S+1) .
$$

Therefore, substituting Eqs. 13, 15, and 16 into Eq. 11 gives

$$
\begin{aligned}
& \sum_{\bar{m}=0} \bar{m}\left\langle 0\left|\mu_{g e}\right| \bar{m}\right\rangle\left\langle\bar{m}\left|\mu_{e g}\right| 0\right\rangle=\mu_{g e}^{2}(0) S+\mu_{g e}(0)\left(\frac{\partial \mu_{g e}}{\partial Q}\right)_{0} \\
& \times \sqrt{\frac{\hbar}{2 m \omega}}(-\delta)+\left(\frac{\partial \mu_{g e}}{\partial Q}\right)_{0}^{2} \frac{\hbar}{2 m \omega}(S+1) .
\end{aligned}
$$

After similar manipulation, we obtain the polarizability to the order of $(\hbar \omega / \Delta)^{2}$ 


$$
\begin{aligned}
\alpha^{\prime}= & \frac{1}{2 k}\left(\frac{\partial \mu_{g g}}{\partial Q}\right)_{0}\left(\frac{\partial \mu_{g g}}{\partial Q}\right)_{0}+\frac{\mu_{g e}^{2}(0)}{\Delta}+\frac{1}{\Delta}\left(\frac{\partial \mu_{g e}}{\partial Q}\right)_{0}^{2} \frac{\hbar}{2 m \omega} \\
& +\frac{1}{\Delta}\left\{-\frac{\hbar \omega}{\Delta}\left[\mu_{g e}^{2}(0) S-\mu_{g e}(0)\left(\frac{\partial \mu_{g e}}{\partial Q}\right)_{0}^{d}\right.\right. \\
& \left.+\left(\frac{\partial \mu_{g e}}{\partial Q}\right)_{0}^{2} \frac{\hbar}{2 m \omega}(S+1)\right]+\left(\frac{\hbar \omega}{\Delta}\right)^{2}\left[\mu_{g e}^{2}(0) S(S+1)\right. \\
& -\mu_{g e}(0)\left(\frac{\partial \mu_{g e}}{\partial Q}\right)_{0} d(2 S+1) \\
& \left.\left.+\left(\frac{\partial \mu_{g e}}{\partial Q}\right)_{0}^{2} \frac{\hbar}{2 m \omega}\left(S^{2}+5 S+1\right)\right]+\cdots\right\} .
\end{aligned}
$$

It is recognized in Eq. 18 that the first term is the lattice relaxation expression for the vibrational contribution to the polarizability and the second term is the electronic contribution to the polarizability. If the vibrational frequencies are much smaller than the electronic frequencies $(\hbar \omega \ll \Delta)$, the dominant vibrational contribution to the polarizability originates mainly from the pure vibrational motion. Furthermore, in addition to the lattice relaxation term and the electronic contribution term, the higher-order correction terms to the polarizability are derived, and they are expressed in terms of the Huang-Rhys factor or the displacement of the oscillators $d$. Therefore, these higher-order correction terms describing the vibrational contribution to the polarizability result from the electron-phonon coupling.

\subsection{Static first hyperpolarizability}

The SOS expression for the first hyperpolarizability $\beta$ is given by

$$
\begin{aligned}
\frac{1}{3 !} \beta=\beta^{\prime}= & \sum_{m \neq 0} \sum_{n \neq 0} \frac{\langle 0|\mu| m\rangle\langle m|\mu| n\rangle\langle n|\mu| 0\rangle}{\left(E_{m}-E_{0}\right)\left(E_{n}-E_{0}\right)} \\
& -\langle 0|\mu| 0\rangle \sum_{m \neq 0} \frac{\langle 0|\mu| m\rangle\langle m|\mu| 0\rangle}{\left(E_{m}-E_{0}\right)^{2}} .
\end{aligned}
$$

For the two-level system with a single vibrational mode, the first hyperpolarizability is expressed by

$$
\begin{aligned}
\beta^{\prime}= & \sum_{m \neq 0} \sum_{n \neq 0} \frac{\langle g 0|\mu| g m\rangle\langle g m|\mu| g n\rangle\langle g n|\mu| g 0\rangle}{\left(E_{g m}-E_{g 0}\right)\left(E_{g n}-E_{g 0}\right)} \\
& +\sum_{m \neq 0} \sum_{\bar{n}=0} \frac{\langle g 0|\mu| g m\rangle\langle g m|\mu| e \bar{n}\rangle\langle e \bar{n}|\mu| g 0\rangle}{\left(E_{g m}-E_{g 0}\right)\left(E_{e \bar{n}}-E_{g 0}\right)} \\
& +\sum_{\bar{m}=0} \sum_{n \neq 0} \frac{\langle g 0|\mu| e \bar{m}\rangle\langle e \bar{m}|\mu| g n\rangle\langle g n|\mu| g 0\rangle}{\left(E_{e \bar{m}}-E_{g 0}\right)\left(E_{g n}-E_{g 0}\right)} \\
& +\sum_{\bar{m}=0} \sum_{\bar{n}=0} \frac{\langle g 0|\mu| e \bar{m}\rangle\langle e \bar{m}|\mu| e \bar{n}\rangle\langle e \bar{n}|\mu| g 0\rangle}{\left(E_{e \bar{m}}-E_{g 0}\right)\left(E_{e \bar{n}}-E_{g 0}\right)} \\
& -\langle g 0|\mu| g 0\rangle \sum_{m \neq 0} \frac{\langle g 0|\mu| g m\rangle\langle g m|\mu| g 0\rangle}{\left(E_{g m}-E_{g 0}\right)^{2}} \\
& -\langle g 0|\mu| g 0\rangle \sum_{\bar{m}=0} \frac{\langle g 0|\mu| e \bar{m}\rangle\langle e \bar{m}|\mu| g 0\rangle}{\left(E_{e \bar{m}}-E_{g 0}\right)^{2}} .
\end{aligned}
$$

Through use of Eqs. 4 and 5, we find that the first and fifth terms in Eq. 20 (the pure vibrational terms) are equal to zero. Therefore, there is no pure vibrational contribution to the first hyperpolarizability.

Substituting Eqs. 4 and 5 into the second and third terms in Eq. 20 with only one pure vibrational frequency in the denominators, we obtain

$$
\begin{aligned}
& \frac{\langle 0|Q| 1\rangle}{\hbar \omega}\left(\frac{\partial \mu_{g g}}{\partial Q}\right)_{0} \sum_{\bar{n}=0} \frac{\langle g 1|\mu| e \bar{n}\rangle\langle e \bar{n}|\mu| g 0\rangle}{\Delta+\bar{n} \hbar \omega} \\
& +\frac{\langle 1|Q| 0\rangle}{\hbar \omega}\left(\frac{\partial \mu_{g g}}{\partial Q}\right) \sum_{0 \bar{m}=0} \frac{\langle g 0|\mu| e \bar{m}\rangle\langle e \bar{m}|\mu| g 1\rangle}{\Delta+\bar{m} \hbar \omega} .
\end{aligned}
$$

Since the matrix elements in Eq. 21 are real valued and $\bar{n}$ and $\bar{m}$ are dummy variables, Eq. 21 can be written as

$2 \frac{\langle 0|Q| 1\rangle}{\hbar \omega}\left(\frac{\partial \mu_{g g}}{\partial Q}\right)_{0} \sum_{\bar{n}=0} \frac{\langle g 1|\mu| e \bar{n}\rangle\langle e \bar{n}|\mu| g 0\rangle}{\Delta+\bar{n} \hbar \omega}$.

Expanding the summation in powers of $\hbar \omega / \Delta$, we have

$$
\begin{aligned}
\sum_{\bar{n}=0} \frac{\langle g 1|\mu| e \bar{n}\rangle\langle e \bar{n}|\mu| g 0\rangle}{\Delta+\bar{n} h \omega} & =\frac{1}{\Delta} \sum_{\bar{n}=0}\left[\left\langle 1\left|\mu_{g e}\right| \bar{n}\right\rangle\left\langle\bar{n}\left|\mu_{e g}\right| 0\right\rangle\right. \\
& \left.\times\left(1-\bar{n} \frac{h \omega}{\Delta}+\bar{n}^{2}\left(\frac{h \omega}{\Delta}\right)^{2}-\cdots\right)\right] .
\end{aligned}
$$

Using Eq. 9 and the completeness relationship for the vibrational levels of the excited state, we write the first term in Eq. 23 as

$\sum_{\bar{n}=0}\left\langle 1\left|\mu_{g e}\right| \bar{n}\right\rangle\left\langle\bar{n}\left|\mu_{e g}\right| 0\right\rangle=2 \mu_{g e}(0)\left(\frac{\partial \mu_{g e}}{\partial Q}\right)_{0}\langle 1|Q| 0\rangle$.

Therefore, the first hyperpolarizability to the order of $1 / \Delta$. $(\hbar \omega / \Delta)^{0}$ becomes

$\beta^{\prime} \simeq \frac{1}{\Delta} \frac{4}{\hbar \omega}\left(\frac{\partial \mu_{g g}}{\partial Q}\right)_{0} \mu_{g e}(0)\left(\frac{\partial \mu_{g e}}{\partial Q}\right)_{0}|\langle 0|Q| 1\rangle|^{2}$.

On the other hand, the electronic contribution to the polarizability is $\alpha^{e}=2 \mu_{g e}^{2} / \Delta$. The first derivative of the electronic contribution to the polarizability with respect to the vibrational mode at the minimum of the ground electronic state is given by

$$
\left(\frac{\partial \alpha^{e}}{\partial Q}\right)_{0}=4 \frac{1}{\Delta} \mu_{g e}(0)\left(\frac{\partial \mu_{g e}}{\partial Q}\right)_{0} .
$$

Through Eq. 26, Eq. 25 can be expressed by

$\beta^{\prime} \simeq \frac{1}{2 k}\left(\frac{\partial \mu_{g g}}{\partial Q}\right)_{0}\left(\frac{\partial \alpha^{e}}{\partial Q}\right)_{0}$,

where $k$ is the force constant of the ground vibrational state and $|\langle 0|Q| 1\rangle|^{2}=\hbar / 2 m \omega$ has been used. This equation is the lattice relaxation expression for the vibrational 
contribution to the first hyperpolarizability $\beta$. If the vibrational frequencies are much smaller than the electronic frequencies $(\hbar \omega \ll \Delta)$, Eq. 27 leads to the dominant contribution to the first hyperpolarizability.

We proceed to determine the contribution from the next higher-order terms to the first hyperpolarizability. To consider the terms involving two electronic excitations $\left(\Delta^{2}\right)$ in the denominators, we have to include the second term in Eq. 23 and the fourth and sixth terms in Eq. 20. The fourth and sixth terms in Eq. 20 are given by

$$
\begin{aligned}
& \sum_{\bar{m}=0} \sum_{\bar{n}=0} \frac{\langle g 0|\mu| e \bar{m}\rangle\langle e \bar{m}|\mu| e \bar{n}\rangle\langle e \bar{n}|\mu| g 0\rangle}{(\Delta+\bar{m} \hbar \omega)(\Delta+\bar{n} \hbar \omega)} \\
& -\langle g 0|\mu| g 0\rangle \sum_{\bar{m}=0} \frac{|\langle g 0|\mu| e \bar{m}\rangle|^{2}}{(\Delta+\bar{m} \hbar \omega)^{2}} .
\end{aligned}
$$

We expand the first term in powers of $\hbar \omega / \Delta$

$$
\begin{aligned}
\frac{1}{\Delta^{2}} \sum_{\bar{m}=0} \sum_{\bar{n}=0}\left[\left\langle 0\left|\mu_{g e}\right| \bar{m}\right\rangle\left\langle\bar{m}\left|\mu_{e e}\right| \bar{n}\right\rangle\left\langle\bar{n}\left|\mu_{e g}\right| 0\right\rangle\right. \\
\left.\times\left(1-(\bar{m}+\bar{n}) \frac{\hbar \omega}{\Delta}+\cdots\right)\right] .
\end{aligned}
$$

Keeping Eq. 29 to the order of $1 / \Delta^{2}$, we obtain

$$
\begin{aligned}
& \sum_{\bar{m}=0} \sum_{\bar{n}=0}\left\langle 0\left|\mu_{g e}\right| \bar{m}\right\rangle\left\langle\bar{m}\left|\mu_{e e}\right| \bar{n}\right\rangle\left\langle\bar{n}\left|\mu_{e g}\right| 0\right\rangle=\mu_{g e}^{2}(0) \mu_{e e}(0) \\
& \quad+\left[2 \mu_{g e}(0)\left(\frac{\partial \mu_{g e}}{\partial Q}\right)_{0}\left(\frac{\partial \mu_{e e}}{\partial Q}\right)_{0}+\mu_{e e}(0)\left(\frac{\partial \mu_{g e}}{\partial Q}\right)_{0}^{2}\right]\left\langle 0\left|Q^{2}\right| 0\right\rangle .
\end{aligned}
$$

Similarly, the second term in Eq. 28 is also expanded to the order of $1 / \Delta^{2}$

$$
\begin{aligned}
\sum_{\bar{m}=0} \frac{|\langle g 0|\mu| e \bar{m}\rangle|^{2}}{(\Delta+\bar{m} \hbar \omega)^{2}}= & \frac{1}{\Delta^{2}} \sum_{\bar{m}=0}\left\langle 0\left|\mu_{g e}\right| \bar{m}\right\rangle\left\langle\bar{m}\left|\mu_{e g}\right| 0\right\rangle \\
& \times\left(1-2 \bar{m} \frac{\hbar \omega}{\Delta}+\cdots\right) .
\end{aligned}
$$

Keeping Eq. 31 to the order of $1 / \Delta^{2}$, we have

$\sum_{\bar{m}=0}\left\langle 0\left|\mu_{g e}\right| \bar{m}\right\rangle\left\langle\bar{m}\left|\mu_{e g}\right| 0\right\rangle=\mu_{g e}^{2}(0)+\left(\frac{\partial \mu_{g e}}{\partial Q}\right)_{0}^{2}\left\langle 0\left|Q^{2}\right| 0\right\rangle$.

Hence, Eq. 28 to the order of $1 / \Delta^{2}$ becomes

$$
\begin{aligned}
& \frac{\mu_{e e}(0)-\mu_{g g}(0)}{\Delta^{2}} \mu_{g e}^{2}(0)+\frac{\left\langle 0\left|Q^{2}\right| 0\right\rangle}{\Delta^{2}} \\
& \times\left[2 \mu_{g e}(0)\left(\frac{\partial \mu_{g e}}{\partial Q}\right)_{0}\left(\frac{\partial \mu_{e e}}{\partial Q}\right)_{0}+\left(\mu_{e e}(0)-\mu_{g g}(0)\right)\left(\frac{\partial \mu_{g e}}{\partial Q}\right)_{0}^{2}\right] .
\end{aligned}
$$

Additionally, the second term in Eq. 23 is given by $-\frac{\hbar \omega}{\Delta^{2}} \sum_{\bar{n}=0} \bar{n}\left\langle 1\left|\mu_{g e}\right| \bar{n}\right\rangle\left\langle\bar{n}\left|\mu_{e g}\right| 0\right\rangle$

substituting Eq. 9 into Eq. 34, we obtain

$$
\begin{gathered}
\sum_{\bar{n}=0} \bar{n}\left[\mu_{g e}^{2}(0)\langle 1 \mid \bar{n}\rangle\langle\bar{n} \mid 0\rangle+\mu_{g e}(0)\left(\frac{\partial \mu_{g e}}{\partial Q}\right)_{0}(\langle 1 \mid \bar{n}\rangle\langle\bar{n}|Q| 0\rangle\right. \\
\left.+\langle 1|Q| \bar{n}\rangle\langle\bar{n} \mid 0\rangle)\left(\frac{\partial \mu_{g e}}{\partial Q}\right)_{0}^{2}\langle 1|Q| \bar{n}\rangle\langle\bar{n}|Q| 0\rangle\right] .
\end{gathered}
$$

After similar manipulation, Eq. 34 can be simplified by

$$
\begin{gathered}
\sum_{\bar{n}=0} \bar{n}\left\langle 1\left|\mu_{g e}\right| \bar{n}\right\rangle\left\langle\bar{n}\left|\mu_{e g}\right| 0\right\rangle=\mu_{g e}^{2}(0)\left(-\frac{\delta}{2}\right)+\mu_{g e}(0)\left(\frac{\partial \mu_{g e}}{\partial Q}\right)_{0} \\
\quad \times \sqrt{\frac{\hbar}{2 m \omega}}(2 S+1)+\left(\frac{\partial \mu_{g e}}{\partial Q}\right)_{0}^{2} \frac{\hbar}{2 m \omega}\left(-\frac{3}{2} \delta\right) .
\end{gathered}
$$

Those terms with the order of $1 / \Delta^{2}$ in Eq. 22 are then given by

$$
\begin{aligned}
& 2 \frac{\langle 0|Q| 1\rangle}{\hbar \omega}\left(\frac{\partial \mu_{g g}}{\partial Q}\right)_{0}\left(-\frac{\hbar \omega}{\Delta^{2}}\right) \\
& \times\left[\mu_{g e}^{2}(0)\left(-\frac{\delta}{2}\right)+\mu_{g e}(0)\left(\frac{\partial \mu_{g e}}{\partial Q}\right)_{0} \sqrt{\frac{\hbar}{2 m \omega}}(2 S+1)\right. \\
& \left.+\left(\frac{\partial \mu_{g e}}{\partial Q}\right)_{0}^{2} \frac{\hbar}{2 m \omega}\left(-\frac{3}{2} \delta\right)\right] .
\end{aligned}
$$

Combining Eqs. 27, 33, and 37, we write the first hyperpolarizability as

$$
\begin{aligned}
\beta^{\prime}= & \frac{1}{2 k}\left(\frac{\partial \mu_{g g}}{\partial Q}\right)_{0}\left(\frac{\partial \alpha^{e}}{\partial Q}\right)_{0}+\frac{\mu_{e e}(0)-\mu_{g g}(0)}{\Delta^{2}} \mu_{g e}^{2}(0) \\
& +\frac{1}{\Delta^{2} 2 m \omega}\left[2 \mu_{g e}(0)\left(\frac{\partial \mu_{g e}}{\partial Q}\right)_{0}\left(\frac{\partial \mu_{e e}}{\partial Q}\right)_{0}\right. \\
& \left.+\left(\mu_{e e}(0)-\mu_{g g}(0)\right)\left(\frac{\partial \mu_{g e}}{\partial Q}\right)_{0}^{2}\right]-\frac{2}{\Delta^{2}} \sqrt{\frac{\hbar}{2 m \omega}}\left(\frac{\partial \mu_{g g}}{\partial Q}\right)_{0} \\
& \times\left[\mu_{g e}^{2}(0)\left(-\frac{\delta}{2}\right)+\mu_{g e}(0)\left(\frac{\partial \mu_{g e}}{\partial Q}\right)_{0} \sqrt{\frac{\hbar}{2 m \omega}}(2 S+1)\right. \\
& \left.+\left(\frac{\partial \mu_{g e}}{\partial Q}\right)_{0}^{2} \frac{\hbar}{2 m \omega}\left(-\frac{3}{2} \delta\right)\right]+\cdots
\end{aligned}
$$

where $\left\langle 0\left|Q^{2}\right| 0\right\rangle=\hbar / 2 m \omega$ and $\langle 0|Q| 1\rangle=\sqrt{\hbar / 2 m \omega}$ have been used.

It is noted that the first term in Eq. 38 is the lattice relaxation expression for vibrational contribution to the first hyperpolarizability. If the vibrational frequencies are much smaller than the electronic frequencies, the lattice relaxation term will dominate. It is also worth mentioning that there is no pure vibrational contribution to the first hyperpolarizability. Therefore, the lattice relaxation contribution with the order of $1 / \Delta$ to the first hyperpolarizability results 
from the coupled motion of the electronic excitation and the nuclear vibration within the adiabatic approximation. Moreover, we also obtain the higher-order correction terms to the first hyperpolarizability.

\subsection{Static second hyperpolarizability}

From the perturbation theory, the second hyperpolarizability $\gamma$ can be expressed by

$$
\begin{aligned}
\frac{1}{4 !} \gamma= & \gamma^{\prime}=\sum_{k \neq 0} \sum_{m \neq 0} \sum_{n \neq 0} \frac{\langle 0|\mu| k\rangle\langle k|\bar{\mu}| m\rangle\langle m|\bar{\mu}| n\rangle\langle n|\mu| 0\rangle}{\left(E_{k}-E_{0}\right)\left(E_{m}-E_{0}\right)\left(E_{n}-E_{0}\right)} \\
& -\sum_{k \neq 0} \frac{\langle 0|\mu| k\rangle\langle k|\mu| 0\rangle}{\left(E_{k}-E_{0}\right)} \sum_{m \neq 0} \frac{\langle 0|\mu| m\rangle\langle m|\mu| 0\rangle}{\left(E_{m}-E_{0}\right)^{2}},
\end{aligned}
$$

where $\bar{\mu}=\mu-\langle 0|\mu| 0\rangle$.

We first consider the pure vibrational terms in Eq. 39 given by

$$
\begin{aligned}
& \sum_{k \neq 0} \sum_{m \neq 0} \sum_{n \neq 0} \frac{\langle g 0|\mu| g k\rangle\langle g k|\bar{\mu}| g m\rangle\langle g m|\bar{\mu}| g n\rangle\langle g n|\mu| g 0\rangle}{\left(E_{g k}-E_{g 0}\right)\left(E_{g m}-E_{g 0}\right)\left(E_{g n}-E_{g 0}\right)} \\
& \quad-\sum_{k \neq 0} \frac{\langle g 0|\mu| g k\rangle\langle g k|\mu| g 0\rangle}{\left(E_{g k}-E_{g 0}\right)} \sum_{m \neq 0} \frac{\langle g 0|\mu| g m\rangle\langle g m|\mu| g 0\rangle}{\left(E_{g m}-E_{g 0}\right)^{2}} .
\end{aligned}
$$

Under the same approximation, Eq. 40 then becomes

$$
\begin{gathered}
\frac{1}{2(\hbar \omega)^{3}}\left(\frac{\partial \mu_{g g}}{\partial Q}\right)_{0}^{4}|\langle 0|Q| 1\rangle|^{2}|\langle 1|Q| 2\rangle|^{2} \\
-\frac{1}{(\hbar \omega)^{3}}\left[\left(\frac{\partial \mu_{g g}}{\partial Q}\right)_{0}^{2}|\langle 0|Q| 1\rangle|^{2}\right]^{2} .
\end{gathered}
$$

Using $|\langle 0|Q| 1\rangle|^{2}=\hbar / 2 m \omega$ and $|\langle 1|Q| 2\rangle|^{2}=\hbar / m \omega$, we find that Eq. 41 is equal to zero. Analogous to the case for the first hyperpolarizability $\beta$, there is also no pure vibrational contribution to the second hyperpolarizability $\gamma$. In particular, as shown in Eq. 18, only the polarizability $\alpha$ contains a pure vibrational contribution.

Next, we consider those terms with two pure vibrational frequencies in the denominators

$$
\begin{aligned}
& \sum_{\bar{k}=0} \sum_{m \neq 0} \sum_{n \neq 0} \frac{\langle g 0|\mu| e \bar{k}\rangle\langle e \bar{k}|\bar{\mu}| g m\rangle\langle g m|\bar{\mu}| g n\rangle\langle g n|\mu| g 0\rangle}{\left(E_{e \bar{k}}-E_{g 0}\right)\left(E_{g m}-E_{g 0}\right)\left(E_{g n}-E_{g 0}\right)} \\
& \quad+\sum_{k \neq 0} \sum_{\bar{m}=0} \sum_{n \neq 0} \frac{\langle g 0|\mu| g k\rangle\langle g k|\bar{\mu}| e \bar{m}\rangle\langle e \bar{m}|\bar{\mu}| g n\rangle\langle g n|\mu| g 0\rangle}{\left(E_{g k}-E_{g 0}\right)\left(E_{e \bar{m}}-E_{g 0}\right)\left(E_{g n}-E_{g 0}\right)} \\
& \quad+\sum_{k \neq 0} \sum_{m \neq 0} \sum_{\bar{n}=0} \frac{\langle g 0|\mu| g k\rangle\langle g k|\bar{\mu}| g m\rangle\langle g m|\bar{\mu}| e \bar{n}\rangle\langle e \bar{n}|\mu| g 0\rangle}{\left(E_{g k}-E_{g 0}\right)\left(E_{g m}-E_{g 0}\right)\left(E_{e \bar{n}}-E_{g 0}\right)} \\
& -\sum_{\bar{k}=0} \frac{\langle g 0|\mu| e \bar{k}\rangle\langle e \bar{k}|\mu| g 0\rangle}{\left(E_{e \bar{k}}-E_{g 0}\right)} \sum_{m \neq 0} \frac{\langle g 0|\mu| g m\rangle\langle g m|\mu| g 0\rangle}{\left(E_{g m}-E_{g 0}\right)^{2}} .
\end{aligned}
$$

Under the same approximation, Eq. 42 can be reduced to

$$
\begin{aligned}
& \frac{\langle g 2|\bar{\mu}| g 1\rangle\langle g 1|\mu| g 0\rangle}{(\hbar \omega)^{2}} \sum_{\bar{k}=0} \frac{\langle g 0|\mu| e \bar{k}\rangle\langle e \bar{k}|\bar{\mu}| g 2\rangle}{(\Delta+\bar{k} \hbar \omega)} \\
& +\frac{\langle g 0|\mu| g 1\rangle\langle g 1|\mu| g 0\rangle}{(\hbar \omega)^{2}} \sum_{\bar{m}=0} \frac{\langle g 1|\bar{\mu}| e \bar{m}\rangle\langle e \bar{m}|\bar{\mu}| g 1\rangle}{(\Delta+\bar{m} \hbar \omega)} \\
& -\frac{|\langle g 0|\mu| g 1\rangle|^{2}}{(\hbar \omega)^{2}} \sum_{\bar{k}=0} \frac{\langle g 0|\mu| e \bar{k}\rangle\langle e \bar{k}|\mu| g 0\rangle}{(\Delta+\bar{k} \hbar \omega)} .
\end{aligned}
$$

We expand the summations in Eq. 43 in terms of $\hbar \omega / \Delta$

$$
\begin{aligned}
\sum_{\bar{k}=0} \frac{\langle g 0|\mu| e \bar{k}\rangle\langle e \bar{k}|\bar{\mu}| g 2\rangle}{(\Delta+\bar{k} \hbar \omega)}= & \frac{1}{\Delta} \sum_{\bar{k}=0}\left[\left\langle 0\left|\mu_{g e}\right| \bar{k}\right\rangle\left\langle\bar{k}\left|\mu_{e g}\right| 2\right\rangle\right. \\
& \left.\times\left(1-\bar{k} \frac{\hbar \omega}{\Delta}+\cdots\right)\right]
\end{aligned}
$$

$\sum_{\bar{m}=0} \frac{\langle g 1|\bar{\mu}| e \bar{m}\rangle\langle e \bar{m}|\bar{\mu}| g 1\rangle}{(\Delta+\bar{m} \hbar \omega)}=\frac{1}{\Delta} \sum_{\bar{m}=0}\left[\left\langle 1\left|\mu_{g e}\right| \bar{m}\right\rangle\left\langle\bar{m}\left|\mu_{e g}\right| 1\right\rangle\right.$

$$
\begin{aligned}
& \left.\times\left(1-\bar{m} \frac{\hbar \omega}{\Delta}+\cdots\right)\right] \\
\sum_{\bar{k}=0} \frac{\langle g 0|\mu| e \bar{k}\rangle\langle e \bar{k}|\mu| g 0\rangle}{(\Delta+\bar{k} \hbar \omega)}= & \frac{1}{\Delta} \sum_{\bar{k}=0}\left[\left\langle 0\left|\mu_{g e}\right| \bar{k}\right\rangle\left\langle\bar{k}\left|\mu_{e g}\right| 0\right\rangle\right. \\
& \left.\times\left(1-\bar{k} \frac{\hbar \omega}{\Delta}+\cdots\right)\right] .
\end{aligned}
$$

The first terms on the right side of Eqs. 44, 45, and 46 become

$\sum_{\bar{k}=0}\left\langle 0\left|\mu_{g e}\right| \bar{k}\right\rangle\left\langle\bar{k}\left|\mu_{e g}\right| 2\right\rangle=\left(\frac{\partial \mu_{g e}}{\partial Q}\right)_{0}^{2}\left\langle 0\left|Q^{2}\right| 2\right\rangle$
$\sum_{\bar{m}=0}\left\langle 1\left|\mu_{g e}\right| \bar{m}\right\rangle\left\langle\bar{m}\left|\mu_{e g}\right| 1\right\rangle=\mu_{g e}^{2}(0)+\left(\frac{\partial \mu_{g e}}{\partial Q}\right)_{0}^{2}\left\langle 1\left|Q^{2}\right| 1\right\rangle$

$\sum_{\bar{k}=0}\left\langle 0\left|\mu_{g e}\right| \bar{k}\right\rangle\left\langle\bar{k}\left|\mu_{e g}\right| 0\right\rangle=\mu_{g e}^{2}(0)+\left(\frac{\partial \mu_{g e}}{\partial Q}\right)_{0}^{2}\left\langle 0\left|Q^{2}\right| 0\right\rangle$.

Therefore, Eq. 43 expanded to the order of $1 / \Delta$ is given by

$\frac{1}{k^{2}}\left(\frac{\partial \mu_{g g}}{\partial Q}\right)_{0}^{2}\left(\frac{\partial \mu_{g e}}{\partial Q}\right)_{0}^{2} \frac{1}{\Delta}$

where $k$ is the force constant of the ground vibrational state. If the vibrational frequencies are much smaller than the electronic frequencies, this term will dominate.

We then consider those terms involved in $\Delta^{2}$ in the denominators, and include both the second terms in Eqs. 44, 45, and 46 and those terms with only one pure vibrational frequency in the denominators in Eq. 39 given by 


$$
\begin{aligned}
& \sum_{k \neq 0} \sum_{\bar{m}=0} \sum_{\bar{n}=0} \frac{\langle g 0|\mu| g k\rangle\langle g k|\bar{\mu}| e \bar{m}\rangle\langle e \bar{m}|\bar{\mu}| e \bar{n}\rangle\langle e \bar{n}|\mu| g 0\rangle}{\left(E_{g k}-E_{g 0}\right)\left(E_{e \bar{m}}-E_{g 0}\right)\left(E_{e \bar{n}}-E_{g 0}\right)} \\
& \quad+\sum_{\bar{k}=0} \sum_{m \neq 0} \sum_{\bar{n}=0} \frac{\langle g 0|\mu| e \bar{k}\rangle\langle e \bar{k}|\bar{\mu}| g m\rangle\langle g m|\bar{\mu}| e \bar{n}\rangle\langle e \bar{n}|\mu| g 0\rangle}{\left(E_{e \bar{k}}-E_{g 0}\right)\left(E_{g m}-E_{g 0}\right)\left(E_{e \bar{n}}-E_{g 0}\right)} \\
& \quad+\sum_{\bar{k}=0} \sum_{\bar{m}=0} \sum_{n \neq 0} \frac{\langle g 0|\mu| e \bar{k}\rangle\langle e \bar{k}|\bar{\mu}| e \bar{m}\rangle\langle e \bar{m}|\bar{\mu}| g n\rangle\langle g n|\mu| g 0\rangle}{\left(E_{e \bar{k}}-E_{g 0}\right)\left(E_{e \bar{m}}-E_{g 0}\right)\left(E_{g n}-E_{g 0}\right)} \\
& \quad-\sum_{k \neq 0} \frac{\langle g 0|\mu| g k\rangle\langle g k|\mu| g 0\rangle}{\left(E_{g k}-E_{g 0}\right)} \sum_{\bar{m}=0} \frac{\langle g 0|\mu| e \bar{m}\rangle\langle e \bar{m}|\mu| g 0\rangle}{\left(E_{e \bar{m}}-E_{g 0}\right)^{2}} .
\end{aligned}
$$

It is noted that $k$ and $n$ are dummy variables and $k$ is only equal to 1 through Eq. 5. Hence, Eq. 51 can be simplified by

$$
\begin{aligned}
& 2 \frac{\langle g 0|\mu| g 1\rangle}{\hbar \omega} \sum_{\bar{m}=0} \sum_{\bar{n}=0} \frac{\langle g 1|\bar{\mu}| e \bar{m}\rangle\langle e \bar{m}|\bar{\mu}| e \bar{n}\rangle\langle e \bar{n}|\mu| g 0\rangle}{(\Delta+\bar{m} \hbar \omega)(\Delta+\bar{n} \hbar \omega)} \\
& \quad+\sum_{\bar{k}=0} \sum_{m \neq 0} \sum_{\bar{n}=0} \frac{\langle g 0|\mu| e \bar{k}\rangle\langle e \bar{k}|\bar{\mu}| g m\rangle\langle g m|\bar{\mu}| e \bar{n}\rangle\langle e \bar{n}|\mu| g 0\rangle}{(\Delta+\bar{k} \hbar \omega)(m \hbar \omega)(\Delta+\bar{n} \hbar \omega)} \\
& \quad-\frac{|\langle g 0|\mu| g 1\rangle|^{2}}{\hbar \omega} \sum_{\bar{m}=0} \frac{\langle g 0|\mu| e \bar{m}\rangle\langle e \bar{m}|\mu| g 0\rangle}{(\Delta+\bar{m} \hbar \omega)^{2}}
\end{aligned}
$$

We expand the summations in terms of $\hbar \omega / \Delta$ to the order of $1 / \Delta^{2}$. Through similar manipulation, Eq. 52 can be expressed by

$$
\begin{aligned}
\frac{1}{\Delta^{2}} & \left\{\frac{1}{k} 2\left(\mu_{e e}(0)-\mu_{g g}(0)\right) \mu_{g e}(0)\left(\frac{\partial \mu_{g e}}{\partial Q}\right)_{0}\left(\frac{\partial \mu_{g g}}{\partial Q}\right)_{0}\right. \\
& +\frac{1}{k} \mu_{g e}^{2}(0)\left(\frac{\partial \mu_{e e}}{\partial Q}\right)_{0}\left(\frac{\partial \mu_{g g}}{\partial Q}\right)_{0} \\
& +\frac{13 \hbar \omega}{k^{2} 2}\left(\frac{\partial \mu_{g e}}{\partial Q}\right)_{0}^{2}\left(\frac{\partial \mu_{e e}}{\partial Q}\right)_{0}\left(\frac{\partial \mu_{g g}}{\partial Q}\right)_{0} \\
& +\frac{1}{k} 2 \mu_{g e}^{2}(0)\left(\frac{\partial \mu_{g e}}{\partial Q}\right)_{0}^{2}+\frac{1 \hbar \omega}{2 k^{2} 2}\left(\frac{\partial \mu_{g e}}{\partial Q}\right)_{0}^{4} \\
& \left.-\frac{1}{2 k} \mu_{g e}^{2}(0)\left(\frac{\partial \mu_{g g}}{\partial Q}\right)_{0}^{2}+\frac{1 \hbar \omega}{2 k^{2} 2}\left(\frac{\partial \mu_{g e}}{\partial Q}\right)_{0}^{2}\left(\frac{\partial \mu_{g g}}{\partial Q}\right)_{0}^{2}\right\},
\end{aligned}
$$

where $k$ is the force constant of the ground vibrational state.

Furthermore, we also have to include the second terms in Eqs. 44, 45, and 46. After similar manipulation to simplify these terms, those terms with the order of $1 / \Delta^{2}$ in Eq. 43 become

$$
\begin{gathered}
-\frac{1}{\Delta^{2}} \frac{1}{2 k}\left(\frac{\partial \mu_{g g}}{\partial Q}\right)_{0}^{2}\left[\mu_{g e}^{2}(0)-\mu_{g e}(0)\left(\frac{\partial \mu_{g e}}{\partial Q}\right)_{0} \sqrt{\frac{\hbar}{2 m \omega}} 4 \delta\right. \\
\left.+\left(\frac{\partial \mu_{g e}}{\partial Q}\right)_{0}^{2} \frac{\hbar}{2 m \omega}(4 S+5)\right] .
\end{gathered}
$$

Collecting all the terms with the order of $1 / \Delta^{2}$ in Eqs. 53 and 54 , we obtain

$$
\begin{aligned}
\frac{1}{k} & \frac{1}{\Delta^{2}} 2\left(\mu_{e e}(0)-\mu_{g g}(0)\right) \mu_{g e}(0)\left(\frac{\partial \mu_{g e}}{\partial Q}\right)_{0}\left(\frac{\partial \mu_{g g}}{\partial Q}\right)_{0} \\
& +\frac{1}{k} \frac{1}{\Delta^{2}} \mu_{g e}^{2}(0)\left(\frac{\partial \mu_{e e}}{\partial Q}\right)_{0}\left(\frac{\partial \mu_{g g}}{\partial Q}\right)_{0}+\frac{1}{k} \frac{1}{\Delta^{2}} 2 \mu_{g e}^{2}(0)\left(\frac{\partial \mu_{g e}}{\partial Q}\right)_{0}^{2} \\
& -\frac{1}{k} \frac{1}{\Delta^{2}} \mu_{g e}^{2}(0)\left(\frac{\partial \mu_{g g}}{\partial Q}\right)_{0}^{2}-\frac{2}{k^{2}} \frac{1 \hbar \omega}{\Delta^{2} 2}\left(\frac{\partial \mu_{g e}}{\partial Q}\right)_{0}^{2}\left(\frac{\partial \mu_{g g}}{\partial Q}\right)_{0}^{2}(S+1) \\
& +\frac{1}{2 k^{2}} \frac{1 \hbar \omega}{\Delta^{2}}\left(\frac{\partial \mu_{g e}}{\partial Q}\right)_{0}^{4}+\frac{1}{k^{2}} \frac{13 \hbar \omega}{\Delta^{2}}\left(\frac{\partial \mu_{g e}}{\partial Q}\right)_{0}^{2}\left(\frac{\partial \mu_{e e}}{\partial Q}\right)_{0}\left(\frac{\partial \mu_{g g}}{\partial Q}\right)_{0} \\
& +\frac{1}{2 k} \frac{1}{\Delta^{2}}\left(\frac{\partial \mu_{g g}}{\partial Q}\right)_{0}^{2} \mu_{g e}(0)\left(\frac{\partial \mu_{g e}}{\partial Q}\right)_{0} \sqrt{\frac{\hbar}{2 m \omega}} 4 \delta .
\end{aligned}
$$

It is worth noting that one half of the contribution from the forth term,

$-\frac{1}{k} \frac{1}{\Delta^{2}} \mu_{g e}^{2}(0)\left(\frac{\partial \mu_{g g}}{\partial Q}\right)_{0}^{2}$,

results from those terms with only one pure vibrational frequency in the denominators in Eq. 51 and the other half of the contribution results from those terms with two pure vibrational frequencies in the denominators in Eq. 42. On the other hand, the electronic contribution to the first hyperpolarizability is

$\beta^{e}=6\left(\mu_{e e}-\mu_{g g}\right) \frac{\mu_{g e}^{2}}{\Delta^{2}}$.

The first derivative of the electrical contribution to the first hyperpolarizability with respect to the vibrational mode at the minimum of the ground electronic state is given by

$$
\begin{aligned}
\left(\frac{\partial \beta^{e}}{\partial Q}\right)_{0}= & 6\left[\left(\frac{\partial \mu_{e e}}{\partial Q}\right)_{0}-\left(\frac{\partial \mu_{g g}}{\partial Q}\right)_{0}\right] \frac{\mu_{g e}^{2}(0)}{\Delta^{2}} \\
& +6\left(\mu_{e e}(0)-\mu_{g g}(0)\right) \frac{2 \mu_{g e}(0)}{\Delta^{2}}\left(\frac{\partial \mu_{g e}}{\partial Q}\right)_{0} .
\end{aligned}
$$

Through Eqs. 58 and 26, the first four terms in Eq. 55 can be expressed by

$\frac{1}{k}\left[\frac{1}{8}\left(\frac{\partial \alpha^{e}}{\partial Q}\right)_{0}\left(\frac{\partial \alpha^{e}}{\partial Q}\right)_{0}+\frac{1}{6}\left(\frac{\partial \mu_{g g}}{\partial Q}\right)_{0}\left(\frac{\partial \beta^{e}}{\partial Q}\right)_{0}\right]$.

Hence, combining Eq. 50 with Eq. 55, we write the second hyperpolarizability as

$$
\begin{aligned}
\gamma^{\prime}= & \frac{1}{\Delta} \frac{1}{k^{2}}\left(\frac{\partial \mu_{g g}}{\partial Q}\right)_{0}^{2}\left(\frac{\partial \mu_{g e}}{\partial Q}\right)_{0}^{2}+\frac{1}{k}\left[\frac{1}{8}\left(\frac{\partial \alpha^{e}}{\partial Q}\right)_{0}\left(\frac{\partial \alpha^{e}}{\partial Q}\right)_{0}\right. \\
& \left.+\frac{1}{6}\left(\frac{\partial \mu_{g g}}{\partial Q}\right)_{0}\left(\frac{\partial \beta^{e}}{\partial Q}\right)_{0}\right]-\frac{1}{\Delta^{2}} \frac{\hbar \omega}{k^{2}}\left(\frac{\partial \mu_{g g}}{\partial Q}\right)_{0}^{2}\left(\frac{\partial \mu_{g e}}{\partial Q}\right)_{0}^{2}(S+1) \\
& +\frac{1}{\Delta^{2}} \frac{\hbar \omega}{4 k^{2}}\left(\frac{\partial \mu_{g e}}{\partial Q}\right)_{0}^{4}+\frac{1}{\Delta^{2}} \frac{3 \hbar \omega}{2 k^{2}}\left(\frac{\partial \mu_{g e}}{\partial Q}\right)_{0}^{2}\left(\frac{\partial \mu_{e e}}{\partial Q}\right)_{0}\left(\frac{\partial \mu_{g g}}{\partial Q}\right)_{0} \\
& +\frac{1}{\Delta^{2}} \frac{1}{2 k}\left(\frac{\partial \mu_{g g}}{\partial Q}\right)_{0}^{2} \mu_{g e}(0)\left(\frac{\partial \mu_{g e}}{\partial Q}\right)_{0} \sqrt{\frac{\hbar}{2 m \omega}} 4 \delta+\cdots
\end{aligned}
$$

If the vibrational frequencies are much smaller than the electronic frequencies, the first term will dominate. The 
second and third terms in Eq. 60 related to the derivatives of the electrical contribution to the polarizability and the first hyperpolarizability are the lattice relaxation expression for the vibrational contribution to the second hyperpolarizability. Moreover, there is no pure vibrational contribution to the second hyperpolarizability. Therefore, the contribution with the order of $1 / \Delta$ to the second hyperpolarizability arises from the coupled motion of the electronic excitation and the nuclear vibration within the adiabatic approximation. In addition, the higher-order correction terms are also obtained.

For the current study, we will focus on a centrosymmetric system with only two electronic energy levels. For this system, the dipole moments are equal to zero, $\mu_{g g}(Q)=\mu_{e e}(Q)=0$. It is straightforward but laborious to derive the second hyperpolarizability to the order of $\Delta^{-4}$ expressed by

$$
\begin{aligned}
\gamma^{\prime}= & \frac{1}{\Delta^{2}}\left[\frac{2 \mu_{g e}^{2}(0)}{k}\left(\frac{\partial \mu_{g e}}{\partial Q}\right)_{0}^{2}+\frac{\hbar \omega}{4 k^{2}}\left(\frac{\partial \mu_{g e}}{\partial Q}\right)_{0}^{4}\right] \\
& -\frac{1}{\Delta^{3}}\left[\mu_{g e}^{4}(0)+\mu_{g e}^{3}(0)\left(\frac{\partial \mu_{g e}}{\partial Q}\right)_{0} \sqrt{\frac{\hbar}{2 m \omega}}(-2 \delta)\right. \\
& +\mu_{g e}^{2}(0)\left(\frac{\partial \mu_{g e}}{\partial Q}\right)_{0}^{2} \frac{\hbar}{2 m \omega}(8 S+6) \\
& +\mu_{g e}(0)\left(\frac{\partial \mu_{g e}}{\partial Q}\right)_{0}^{3}\left(\frac{\hbar}{2 m \omega}\right)^{3 / 2}(-8 \delta) \\
& \left.+\left(\frac{\partial \mu_{g e}}{\partial Q}\right)_{0}^{4}\left(\frac{\hbar}{2 m \omega}\right)^{2}(2 S+3)\right]+\frac{\hbar \omega}{\Delta^{4}}\left[\mu_{g e}^{4}(0) 4 S\right. \\
& +\mu_{g e}^{3}(0)\left(\frac{\partial \mu_{g e}}{\partial Q}\right)_{0} \sqrt{\frac{\hbar}{2 m \omega}}\left[-\left(6 \delta+\frac{3}{2} \delta^{3}\right)\right] \\
& +\mu_{g e}^{2}(0)\left(\frac{\partial \mu_{g e}}{\partial Q}\right)_{0}^{2} \frac{\hbar}{2 m \omega}\left(12 S^{2}+56 S+8\right) \\
& +\mu_{g e}(0)\left(\frac{\partial \mu_{g e}}{\partial Q}\right)_{0}^{3}\left(\frac{\hbar}{2 m \omega}\right)^{3 / 2}\left[-\left(30 \delta+7 \delta^{3}\right)\right] \\
& \left.+\left(\frac{\partial \mu_{g e}}{\partial Q}\right)_{0}^{4}\left(\frac{\hbar}{2 m \omega}\right)^{2}\left(5 S^{2}+49 S+8\right)\right]+\cdots
\end{aligned}
$$

\section{Model calculations}

\subsection{Two-level system: ethylene}

It is important to compare the relative importance of the various contributions to the polarizability and the hyperpolarizabilities. To obtain an order-of-magnitude estimate for those terms derived in the previous sections, we first consider the simplest $\pi$-electron system: ethylene. We will use Yaron and Silbey's parameters for ethylene [3]. A typical vibrational frequency $\omega$ for a $\mathrm{C}=\mathrm{C}$ double bond is
$0.19 \mathrm{eV}$. The energy difference between the ground and excited electronic states $\Delta$ is taken to be the band gap in polyacetylene, approximately $1.4 \mathrm{eV}$. The average vibrational amplitude $\sqrt{\hbar / 2 m \omega}$ for a $\mathrm{C}=\mathrm{C}$ double bond is $0.043 \AA$. The calculated change in bond length on excitation of octatetraene is of the same order of magnitude $0.059 \AA$ for the central bonds, so we expect $\delta$ to be of order unity.

Furthermore, in order to estimate the derivative of the transition moment, we will also follow Yaron and Silbey's argument [3] that the transition moment is just proportional to the bond length

$$
\left(\frac{\partial \mu_{g e}}{\partial Q}\right)_{0}=\frac{\mu_{g e}(0)}{Q_{e}}
$$

where $Q_{e}$ is the bond length $\left(Q_{e}=1.4 \AA\right)$. Therefore, substituting these parameters into Eqs. 18 and 61, we can estimate the relative magnitude of various terms in the polarizability and in the second hyperpolarizability. These results are shown in Tables 1 and 2, respectively.

Table 1 presents the magnitude of various contributions from those terms in Eq. 18 relative to the electronic contribution $\mu_{g e}^{2}(0) / \Delta$. As shown in Table 1 , the relative magnitude of these contributions decreases with the increasing of the order of $\hbar \omega / \Delta$, and it also decreases with the increasing of the order of $\left(\partial \mu_{g e} / \partial Q\right)_{0}$. In addition, the sum of the relative magnitude from the vibrational contribution terms is -0.023311 . Therefore, the contributions of these higher-order terms to the polarizability for ethylene are extremely small.

Table 2 presents the relative magnitude of various contributions from those terms in Eq. 61. The value of the contribution from the $\alpha^{2}$ term $\left(\mu_{g e}^{2}(0) / \Delta^{2}\right)\left(\partial \mu_{g e} / \partial Q\right)_{0}^{2}$ relative to the electronic contribution $\mu_{g e}^{4}(0) / \Delta^{3}$ is the same as that obtained by Yaron and Silbey [3]. However, the value of the contribution from the term $\mu_{g e}^{4}(0) / \Delta^{4}$ relative to the electronic contribution is approximately four times as large as that obtained by Yaron and Silbey. This difference results from the fact that Yaron and Silbey only considered those terms with a pure vibrational frequency in the denominator in Eq. 39. Additionally, the sum of the relative magnitude from the vibrational contribution terms is 0.184496. Analogous to the case for the polarizability, Table 2 also shows that the contributions from the higher-

Table 1 Relative magnitude of various contributions to the polarizability $\alpha$ for ethylene

\begin{tabular}{lccc}
\hline$\frac{1}{\Delta}\left(\frac{\hbar \omega}{\Delta}\right)^{n}$ & $\mu_{g e}^{2}(0)$ & $\mu_{g e}(0)\left(\frac{\partial \mu_{g e}}{\partial Q}\right)_{0}$ & $\left(\frac{\partial \mu_{g e}}{\partial Q}\right)_{0}^{2}$ \\
\hline 0 & 1 & 0 & 0.00094 \\
1 & -0.033 & 0.0041 & -0.00016 \\
2 & 0.0056 & -0.00083 & 0.000039 \\
\hline
\end{tabular}


Table 2 Relative magnitude of various contributions to the second hyperpolarizability $\gamma$ for ethylene

\begin{tabular}{lccccr}
\hline$\left(\frac{1}{\Delta}\right)^{n}$ & $\mu_{g e}^{4}(0)$ & $\mu_{g e}^{3}(0)\left(\frac{\partial \mu_{g e}}{\partial Q}\right)_{0}$ & $\mu_{g e}^{2}(0)\left(\frac{\partial \mu_{g e}}{\partial Q}\right)_{0}^{2}$ & $\mu_{g e}(0)\left(\frac{\partial \mu_{g e}}{\partial Q}\right)_{0}^{3}$ & \multicolumn{1}{c}{$\left(\frac{\partial \mu_{g e}}{\partial Q}\right)_{0}^{4}$} \\
\hline 2 & 0 & 0 & 0.028 & 0 & $6.6 \times 10^{-6}$ \\
3 & -1 & 0.061 & -0.0075 & 0.00023 & $-3.1 \times 10^{-6}$ \\
4 & 0.13 & -0.03 & 0.0029 & -0.00014 & $2.5 \times 10^{-6}$ \\
\hline
\end{tabular}

order terms to the second hyperpolarizability for ethylene are relatively small.

As shown in Tables 1 and 2, the vibrational contribution to the polarizability and to the second hyperpolarizability for ethylene is much smaller than the electronic contribution. Most of the correction terms are related to the derivative of the electronic transition moment; hence, the vibrational contribution from these terms originates from the dependence of the electronic transition moment on the vibrational displacements. Equation 62 has been used to estimate the derivative of the electronic transition moment [3], and it was pointed out that the dependence of the electronic transition moment on the vibrational displacements for ethylene is very weak. Therefore, the vibrational contribution from these correction terms is small. Furthermore, it is noted in the present model that the sum of the relative magnitude from the vibrational contribution terms for the polarizability for ethylene is -0.023311 . In contrast with the case for the second hyperpolarizability, the sum of the relative magnitude from the vibrational contribution terms is 0.184496 . Thus, the vibrational contribution for the second hyperpolarizability for ethylene is more significant than that for the polarizability.

\subsection{Two-band system: polyacetylene}

It is significant to consider whether any qualitatively new effects will arise in polymeric systems. The simplest twoband system, polyacetylene, will be examined using the tight-binding approximation with harmonic vibrations. The Longuet-Higgins and Salem's model is used to describe the polyenes [26-29]

$H=\sum_{n} \beta\left(r_{n}\right)\left(c_{n}^{+} c_{n+1}+c_{n+1}^{+} c_{n}\right)+\sum_{n} f\left(r_{n}\right)$,

where $\beta(r)$ is the transfer (resonance) integral and $f(r)$ is $\sigma$ bond compressional energy. Following Kürti and Kuzmany [26], we assume that $\beta(r)$ is given by the exponential function

$\beta(r)=-A_{\beta} e^{-r / B_{\beta}}$,

where $r$ is the distance between the two carbon atoms, $A_{\beta}=243.5 \mathrm{eV}$, and $B_{\beta}=0.3075 \AA$. At equilibrium, the lengths of single and double bonds are $R_{1}=1.45 \AA$ and $R_{2}=1.36 \AA$, respectively. For a perfect dimerized chain with periodic boundary condition, the eigenvalue $\omega_{k}$ for the one electron state $|k\rangle$ is given by

$$
\begin{aligned}
\omega_{k} & =\left[\beta_{1}^{2}+\beta_{2}^{2}+2 \beta_{1} \beta_{2} \cos (k a)\right]^{1 / 2} \\
& =\sqrt{2} t_{0} \sqrt{\cosh 2 x_{0}+\cos k a},
\end{aligned}
$$

where $a$ is the unit cell length, $t_{0}=A_{\beta} e^{-a / 2 B_{\beta}}, x_{0}=\left(r_{1}-\right.$ $\left.r_{2}\right) / 2 B_{\beta}$, and $\beta_{1}$ and $\beta_{2}$ are the resonance integrals for the single and double bonds, respectively.

According to Yaron and Silbey's argument [3], we only need to consider the optical $K=0$ phonon mode. In order to calculate the polarizability $\alpha$ and the second hyperpolarizability $\gamma$, we will use simple displaced harmonic oscillators to approximate the excited state potential surfaces. Although the derivation is similar to the case for the two-level system, a sum over all excited electronic states is necessary. From Eq. 18, the polarizability with real transition moments is given by

$$
\begin{aligned}
\alpha^{\prime}= & \frac{1}{2 k}\left(\frac{\partial \mu_{g g}}{\partial Q}\right)_{0}\left(\frac{\partial \mu_{g g}}{\partial Q}\right)_{0} \\
& +\sum_{i=1}^{\infty}\left(\frac{\mu_{g e_{i}}^{2}(0)}{\Delta_{i}}+\frac{1}{\Delta_{i}}\left(\frac{\partial \mu_{g e_{i}}}{\partial Q}\right)_{0}^{2} \frac{\hbar}{2 m \omega}\right) \\
& +\sum_{i=1}^{\infty} \frac{1}{\Delta_{i}}\left\{-\frac{\hbar \omega}{\Delta_{i}}\left[\mu_{g e_{i}}^{2}(0) S_{i}-\mu_{g e_{i}}(0)\left(\frac{\partial \mu_{g e_{i}}}{\partial Q}\right)_{0} d_{i}\right.\right. \\
& \left.+\left(\frac{\partial \mu_{g e_{i}}}{\partial Q}\right)_{0}^{2} \frac{\hbar}{2 m \omega}\left(S_{i}+1\right)\right] \\
& +\left(\frac{\hbar \omega}{\Delta_{i}}\right)^{2}\left[\mu_{g e_{i}}^{2}(0) S_{i}\left(S_{i}+1\right)\right. \\
& -\mu_{g e_{i}}(0)\left(\frac{\partial \mu_{g e_{i}}}{\partial Q}\right)_{0}\left(2 S_{i}+1\right) \\
& \left.\left.+\left(\frac{\partial \mu_{g e_{i}}}{\partial Q}\right)_{0}^{2} \frac{\hbar}{2 m \omega}\left(S_{i}^{2}+5 S_{i}+1\right)\right]+\cdots\right\},
\end{aligned}
$$

where $Q$ refers to the $K=0$ phonon mode, $\mu_{g e_{i}}$ is the transition moment between the ground and an excited electronic state, and the sum is over all excited electronic states.

The band theory for polyacetylene within the tight binding approximation has been developed by Cojan, Agrawal, and Flytzanis [28, 29]. For polyacetylene, the dipole moment for the ground electronic state vanishes $\left(\mu_{g g}(Q)=0\right)$, so the first term in Eq. 66 becomes zero. The second term in Eq. 66 is the electronic contribution to the polarizability expressed by 
$\sum_{i=1}^{\infty} \frac{\mu_{g e_{i}}^{2}(0)}{\Delta_{i}}$.

In the large $N$ limit, $\mu_{g e_{i}}$ is replaced by $\Omega(k)$ and the sum over states is replaced by an integral over the first Brillouin zone $[26,28-30]$

$2 N \frac{a}{2 \pi} \int_{-\frac{\pi}{a}}^{\frac{\pi}{a}} \frac{|\Omega(k)|^{2}}{\varepsilon_{k}} \mathrm{~d} k$,

where $\varepsilon_{k}=2 \omega_{k}$ is the transition energy at $k$. A factor of 2 has been included to account for the double occupation of the orbitals. When we derived Eq. 68 from Eq. 67, the summation over many-electron wave functions has been replaced by the summation over single-electron wave functions and the transition matrix element $\mu_{g e_{i}}$ has been replaced by $\Omega(k)$ given by

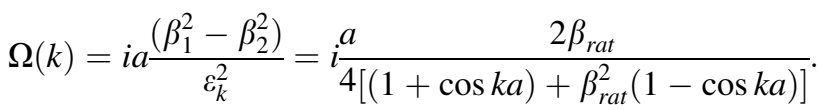

Following Yaron and Silbey [3], we use periodic boundary conditions for the phonon and treat the electronic motion as a open chain. The $K=0$ optical phonon for a chain of $2 \mathrm{~N}$ carbons is

$Q_{K=0}=\frac{1}{\sqrt{2 N}} \sum_{j=1}^{2 N}(-1)^{j} \mathrm{~d} r_{j}$.

Since the electronic wave functions are invariant to the transformation $\left(\beta_{1}, \beta_{2}\right) \Rightarrow\left(c \beta_{1}, c \beta_{2}\right)$ where $c$ is a constant [3], $\mu_{g e_{i}}$ depends only on the ratio

$\beta_{\text {rat }}=\frac{\beta_{1}-\beta_{2}}{\beta_{1}+\beta_{2}}=-\tanh x_{0}$.

Therefore, we can replace the derivative with respect to $Q$ in Eq. 66 with a derivative with respect to $\beta_{\text {rat }}$

$$
\left(\frac{\partial \mu_{g e_{i}}}{\partial Q}\right)_{0}=\left(\frac{\partial \mu_{g e_{i}}}{\partial \beta_{r a t}}\right)_{0}\left(\frac{\partial \beta_{r a t}}{\partial Q}\right)_{0} .
$$

In the large $N$ limit, we have

$$
\begin{aligned}
& \left(\frac{\partial \mu_{g e_{i}}}{\partial Q}\right)_{0} \rightarrow\left(\frac{\mathrm{d} \Omega(k)}{\mathrm{d} Q}\right)_{0}=\left(\frac{\mathrm{d} \Omega(k)}{\mathrm{d} \beta_{r a t}}\right)_{0}\left(\frac{\mathrm{d} \beta_{r a t}}{\mathrm{~d} Q}\right)_{0} \\
& \quad=i \frac{a(1+\cos k a)-\beta_{r a t}^{2}(1-\cos k a)}{2\left[(1+\cos k a)+\beta_{r a t}^{2}(1-\cos k a)\right]^{2}} \sqrt{\frac{1}{2 N B_{\beta} \cosh ^{2} x_{0}}} .
\end{aligned}
$$

Furthermore, the displacement $d_{i}$ is replaced by the electron-phonon coupling $\lambda_{k}$ [31] $d_{i} \rightarrow \lambda_{k}=-\frac{1}{k_{0}}\left(\frac{\partial \varepsilon_{k}(Q)}{\partial Q}\right)_{0}$

where 0 is the equilibrium geometry of the ground electronic state, $k_{0}$ is the force constant for the $K=0$ phonon mode in the ground (and excited) state, and $\varepsilon_{k}$ is the energy of the excited state relative to the ground state. Similarly, the Huang-Rhys factor $S_{i}$ is also expressed by the electron-phonon coupling $\lambda_{k}$

$S_{i}=\frac{1}{2} \frac{k d_{i}^{2}}{\hbar \omega} \rightarrow \frac{1}{2} \frac{k_{0}}{\hbar \omega} \lambda_{k}^{2}$.

In addition, the size dependence of each term in Eq. 66 can be analyzed in the large $N$ limit. It is noted that the electronic contribution in Eq. 68 is proportional to $N$. From Eqs. 72, 73, and 74, we find for polyacetylene that $\left(\partial \mu_{g e_{i}} / \partial Q\right)_{0}$ is proportional to $N^{-1 / 2}, d_{i}$ to $N^{-1 / 2}$, and $S_{i}$ to $N^{-1}$. For example, the size dependence for the third term in Eq. 66 is given by

$\sum_{i=1}^{\infty} \frac{1}{\Delta_{i}}\left(\frac{\partial \mu_{g e_{i}}}{\partial Q}\right)_{0}^{2} \frac{\hbar}{2 m \omega} \sim N\left(\frac{1}{\sqrt{N}}\right)^{2}=1$,

and the size dependence for the fourth term becomes

$\sum_{i=1}^{\infty} \frac{1}{\Delta_{i}}\left(-\frac{\hbar \omega}{\Delta_{i}}\right) \mu_{g e_{i}}^{2}(0) S_{i} \sim N\left(\frac{1}{N}\right)=1$.

Table 3 displays the size dependence of each term in Eq. 66 for the polarizability of polyacetylene in the large $N$ limit.

As shown in Table 3, only the size dependence of the electronic contribution term is proportional to $N$, while the size dependence of the other terms is smaller than $N$. Therefore, these higher-order terms for polyacetylene are negligible and only the electronic contribution is important in the large $N$ limit.

We can obtain the expression for the second hyperpolarizability similar to Eq. 60 to the order of $\Delta^{-2}$. It is noted that $\mu_{g g}(Q)=0$ for polyacetylene, so only two terms survive. Following the derivation of the second hyperpolarizability for the two-level system, we have

$$
\begin{aligned}
\gamma^{\prime}= & \frac{1}{8 k_{0}}\left(\frac{\partial \alpha^{e}}{\partial Q}\right)_{0}\left(\frac{\partial \alpha^{e}}{\partial Q}\right)_{0} \\
& +\frac{\hbar \omega}{4 k_{0}^{2}} \sum_{i=1}^{\infty} \frac{1}{\Delta_{i}}\left(\frac{\partial \mu_{g e_{i}}}{\partial Q}\right)_{0}^{2} \sum_{j=1}^{\infty} \frac{1}{\Delta_{j}}\left(\frac{\partial \mu_{g e_{j}}}{\partial Q}\right)_{0}^{2} .
\end{aligned}
$$

In the large $N$ limit, the derivative of the polarizability with respect to the vibrational mode

$$
\left(\frac{\partial \alpha^{e}}{\partial Q}\right)_{0}=\sum_{i=1}^{\infty} \frac{4}{\Delta_{i}} \mu_{g e_{i}}(0)\left(\frac{\partial \mu_{g e_{i}}}{\partial Q}\right)_{0}
$$


Table 3 The size dependence of each term in Eq. 66 for the polarizability of polyacetylene in the large $N$ limit

\begin{tabular}{lll}
\hline$(1 / 2 k)\left(\partial \mu_{g g} / \partial Q\right)_{0}\left(\partial \mu_{g g} / \partial Q\right)_{0}$ & $=0$ & $=0$ \\
$\sum_{i=1}^{\infty} \mu_{g e_{i}}^{2}(0) / \Delta_{i}$ & $\sim N$ & $=N$ \\
$\sum_{i=1}^{\infty}\left(1 / \Delta_{i}\right)\left(\partial \mu_{g e_{i}} / \partial Q\right)_{0}^{2}(\hbar / 2 m \omega)$ & $\sim N(1 / \sqrt{N})^{2}$ & $=1$ \\
$\sum_{i=1}^{\infty}\left(1 / \Delta_{i}\right)\left(-\hbar \omega / \Delta_{i}\right) \mu_{g e_{i}}^{2}(0) S_{i}$ & $\sim N(1 / N)$ & $=1$ \\
$\sum_{i=1}^{\infty}\left(1 / \Delta_{i}\right)\left(-\hbar \omega / \Delta_{i}\right) \mu_{g e_{i}}(0)\left(\partial \mu_{g e_{i}} / \partial Q\right)_{0}\left(-d_{i}\right)$ & $\sim N(1 / \sqrt{N})^{2}$ & $=1$ \\
$\sum_{i=1}^{\infty}\left(1 / \Delta_{i}\right)\left(-\hbar \omega / \Delta_{i}\right)\left(\partial \mu_{g e_{i}} / \partial Q\right)_{0}^{2}(\hbar / 2 m \omega)\left(S_{i}+1\right)$ & $\sim N(1 / \sqrt{N})^{2}(1 / N+1)$ & $=1 / N+1$ \\
$\sum_{i=1}^{\infty}\left(1 / \Delta_{i}\right)\left(\hbar \omega / \Delta_{i}\right)^{2} \mu_{g e_{i}}^{2}(0) S_{i}\left(S_{i}+1\right)$ & $\sim N(1 / N)(1 / N+1)$ & $=1 / N+1$ \\
$\sum_{i=1}^{\infty}\left(1 / \Delta_{i}\right)\left(\hbar \omega / \Delta_{i}\right)^{2} \mu_{g e_{i}}(0)\left(\partial \mu_{g e_{i}} / \partial Q\right)_{0}\left(-d_{i}\right)\left(2 S_{i}+1\right)$ & $\sim N(1 / \sqrt{N})(1 / \sqrt{N})(1 / N+1)$ & $=1 / N+1$ \\
$\sum_{i=1}^{\infty}\left(1 / \Delta_{i}\right)\left(\hbar \omega / \Delta_{i}\right)^{2}\left(\partial \mu_{g e_{i}} / \partial Q\right)_{0}^{2}(\hbar / 2 m \omega)\left(S_{i}^{2}+5 S_{i}+1\right)$ & $\sim N(1 / \sqrt{N})^{2}\left(1 / N^{2}+1 / N+1\right)$ & $=1 / N^{2}+1 / N+1$ \\
\hline
\end{tabular}

becomes

$2 N \frac{a}{2 \pi} \int_{-\frac{\pi}{a}}^{\frac{\pi}{a}} \frac{4}{\varepsilon_{k}} \Omega^{*}(k)\left(\frac{\mathrm{d} \Omega(k)}{\mathrm{d} Q}\right)_{0} \mathrm{~d} k$.

We use Kürti and Kuzmany's parameters for polyacetylene [26]. The force constant of the $K=0$ phonon mode is 5.390 mdyne/A. Therefore, we can calculate the $\alpha^{2}$ term

$\gamma^{\prime \nu}=\frac{1}{8 k_{0}}\left(\frac{\partial \alpha^{e}}{\partial Q}\right)_{0}\left(\frac{\partial \alpha^{e}}{\partial Q}\right)_{0}$.

It is noticed that the size dependence of the $\alpha^{2}$ term is proportional to $N$. On the other hand, through Eq. 72, the size dependence of the second term in Eq. 77 is given by

$\frac{\hbar \omega}{4 k_{0}^{2}} \sum_{i=1}^{\infty} \frac{1}{\Delta_{i}}\left(\frac{\partial \mu_{g e_{i}}}{\partial Q}\right)_{0}^{2} \times \sum_{j=1}^{\infty} \frac{1}{\Delta_{j}}\left(\frac{\partial \mu_{g e_{j}}}{\partial Q}\right)_{0}^{2} \sim 1$.

Hence, only the $\alpha^{2}$ term contributes to the second hyperpolarizability to the order of $\Delta^{-2}$ in the large $N$ limit.

Furthermore, we compare the contribution of the $\alpha^{2}$ term with the value of the pure electronic second hyperpolarizability. The band theory result for the electronic second hyperpolarizability is given by [29]

$\gamma^{\prime e}=2 N \frac{a}{2 \pi} \int_{-\frac{\pi}{a}}^{\frac{\pi}{a}}\left[\frac{1 \partial}{\varepsilon_{k} \partial k}\left(\frac{\Omega^{*}(k)}{\varepsilon_{k}}\right) \frac{\partial}{\partial k}\left(\frac{\Omega(k)}{\varepsilon_{k}}\right)-\frac{|\Omega(k)|^{4}}{\varepsilon_{k}^{3}}\right] \mathrm{d} k$.

Through Eqs. 80 and 82, we determine the ratio of vibrational contribution from the $\alpha^{2}$ term to electronic contribution, $\gamma^{\prime v} / \gamma^{\prime e}=44 \%$. If we express the total second hyperpolarizability as a sum of the contributions from the $\alpha^{2}$ term and the pure electronic term, $\gamma^{\prime}=\gamma^{\prime} e+\gamma^{\prime}$. Thus, the $\alpha^{2}$ term contributes $31 \%\left(\gamma^{\prime} v / \gamma^{\prime}\right)$ to the whole second hyperpolarizability. The result is different from Yaron and Silbey's result [3]. This inconsistency arises from the fact that we use the different parameters for the transfer integrals and Yaron and Silbey use the parameters for a $\mathrm{C}=\mathrm{C}$ double bond to approximate the force constant $k_{0}$ for polyacetylene.

\section{Discussion and conclusions}

We applied the exact SOS formulas for static polarizabilities and hyperpolarizabilities expressed in terms of vibronic states to the two-level system with a single vibrational mode. For this system, we derived vibrational contributions to (hyper)polarizabilities in a systematic expansion in the powers of $\hbar \omega / \Delta$, and obtained the lattice relaxation expressions for the vibrational contributions and the contributions from the next higher-order correction terms. Additionally, we also obtained additional terms not contained in the formulas obtained using the Placzek's approximation. Those higher-order terms for the vibrational contribution expressed in terms of the Huang-Rhys factor or the displacement of the oscillators depend on the strength of the electron-phonon coupling. Similarly, this method was also generalized to the multi-level system with multiple vibrational modes. By performing calculations on model systems, we found that the contributions from the higher-order terms to the polarizability and to the second hyperpolarizability for ethylene are relatively small. On the other hand, it was shown that only electronic contribution to the polarizability for polyacetylene is significant. Moreover, the contribution from the $\alpha^{2}$ term and the electronic contribution for polyacetylene are comparable. The contribution of the $\alpha^{2}$ term is $44 \%$ of the electronic contribution. Therefore, inclusion of vibrations for polyacetylene has a non-negligible effect on the second hyperpolarizability.

We have examined the validity of the lattice relaxation expression of vibrational contributions to static linear and nonlinear optical coefficients from the exact quantum mechanical expression. If the vibrational frequencies are much smaller than the electronic frequencies, the lattice relaxation expression for vibrational contributions to the polarizability will dominate, and this term is a pure vibrational contribution. In addition, the contributions from the higher-order terms to the polarizability are negligible in the solid-state limit. In particular, there are no pure vibrational contributions to first and the second hyperpolarizabilities. Similarly, the lattice relaxation expression 
for vibrational contributions to the first hyperpolarizability is dominant. However, there exist other terms at the same order as the lattice relaxation expression for vibrational contributions to the second hyperpolarizability. When the dipole moment of the ground electronic state for symmetric modes vanishes, only two terms at this order survive. One is the lattice relaxation expression $\left(\alpha^{2}\right.$ term) and the other involves the derivative of the electronic transition moment with respect to the vibrational mode. In the solid-state limit, only the $\alpha^{2}$ term is important.

The current study concentrates on static vibrational polarizabilities and hyperpolarizabilities determined from the general perturbation approach under the electrical and mechanical harmonicities. Additionally, two special cases, ethylene and polyacetylene, are considered to compare the contributions from various terms to the polarizability and to the second hyperpolarizability. In the future, dynamic polarizabilities and hyperpolarizabilities including the frequency dependence of the electric field will be considered under the present model, and the effects of the correction terms from anharmonicity contributions and the chainlength dependence of the contributions from various terms in polarizabilities and hyperpolarizabilities deserve further investigation.

Acknowledgments We wish to thank the National Science Council, Taiwan for financial support.

\section{References}

1. Chemla DS, Zyss J (eds) (1987) Nonlinear optical properties of organinc molecules and crystals. Academic Press, New York

2. Castiglioni C, Gussoni M, Del Zoppo M, Zerbi G (1992) Solid State Commun 82:13

3. Yaron D, Silbey RJ (1991) J Chem Phys 95:563
4. Bishop DM, Kirtman B (1991) J Chem Phys 95:2646

5. Kirtman B, Bishop DM (1990) Chem Phys Lett 175:601

6. Shelton DP, Ulivi L (1988) J Chem Phys 89:149

7. Bishop DM (1990) Rev Mod Phys 62:343

8. Bishop DM, Luis JM, Kirtman B (1998) J Chem Phys 108:10013

9. Bishop DM, Kirtman B (1992) J Chem Phys 97:5255

10. Bishop DM, Kirtman B, Champagne B (1997) J Chem Phys 107:5780

11. Champagne B, Luis JM, Duran M, Andrês JL, Kirtman B (2000) J Chem Phys 112:1011

12. Luis JM, Duran M, Champagne B, Kirtman B (2000) J Chem Phys 113:5203

13. Torrent-Sucarrat M, Solà M, Duran M, Luis JM, Kirtman B (2003) J Chem Phys 118:711

14. Torrent-Sucarrat M, Luis JM, Kirtman B (2005) J Chem Phys 122:204108

15. Luis JM, Torrent-Sucarrat M, Christiansen O, Kirtman B (2007) J Chem Phys 127:084118

16. Lu D, Chen G, Perry JW, Goddard WA III (1994) J Am Chem Soc 116:10679

17. Castiglioni C, Del Zoppo M, Zerbi G (1996) Phys Rev B 53:13319

18. Bishop DM, Kirtman B (1997) Phys Rev B 56:2273

19. Castiglioni C, Del Zoppo M, Zerbi G (1997) Phys Rev B 56:2275

20. Kim HS, Cho M, Jeon SJ (1997) J Chem Phys 107:1936

21. Bishop DM, Champagne B, Kirtman B (1998) J Chem Phys 109:9987

22. Del Zoppo M, Castiglioni C, Zerbi G, Rui M, Gussoni M (1992) Synth Met 51:135

23. Castiglioni C, Del Zoppo M, Zerbi G (1993) J Raman Spectrosc $24: 485$

24. Ting C (1968) Spectrochim Acta Part A 24:1177

25. Henderson B, Imbusch GF (2006) Optical spectroscopy of inorganic solids. Oxford University Press, Oxford

26. Kürti J, Kuzmany H (1991) Phys Rev B 44:597

27. Longuet-Higgins HC, Salem L (1959) Proc R Soc London Ser A 251:172

28. Cojan C, Agrawal GP, Flytzanis C (1977) Phys Rev B 15:909

29. Agrawal GP, Cojan C, Flytzanis C (1978) Phys Rev B 17:776

30. Horovitz B, Vardeny Z, Ehrenfreund E, Brafman O (1986) J Phys C 19:7291

31. Jin BY, Silbey R (1995) J Chem Phys 102:4251 